\title{
Force Degradation of Orthodontic Elastomeric Chain due to Commonly Consumed Liquids: An in vitro Study
}

\author{
${ }^{1}$ Rameez Asif Nachan, ${ }^{2}$ Ajit Kalia, ${ }^{3}$ Ibrahim Al-Shahrani
}

\begin{abstract}
Introduction: Forces delivered by elastomeric chains are not constant and degrade overtime. The degree of degradation could result in forces that fall below the threshold value, precluding any therapeutic effect. Correct evaluation of degradation of force of these materials under various environmental conditions is the need of hour.
\end{abstract}

Objective: To analyze and compare the effects of commonly consumed liquids, such as Cola, tea and mouthwash, on the force degradation of orthodontic elastomeric chains was conducted.

\begin{abstract}
Materials and methods: An in vitro study was done to assess factors which affect the decay properties of the three elastomeric modules (Ormco Power Chain, 3M Unitek Alastik Chain and American Orthodontics) in the following food stimulants: black tea, milk, aerated drink, mouthwash and artificial saliva. Two different module lengths (long and short) of each product were used for the initial load-comparison study and force decay was evaluated over 21 days at constant extension and at decreasing extension. Means of percentage change in tensile strength were calculated and analyzed using one way analysis of variance (ANOVA) with Tukey's correction for multiple group comparisons. $p<0.05$ was considered to be statistically significant.
\end{abstract}

Results: The evaluated force degradation in percentage in tensile strength on 21 days post-treatment in tea was significantly higher in product $A$ compared to products $B$ and $C$ with product type short. The evaluated force degradation in percentage in tensile strength on 21 days post-treatment in Cola was significantly higher in products $A$ and $C$ as compared to product $B$ with product type short. The force degradation of product $B$ was significantly less compared to the products $A$ and $C$ initially. However, it degrades at the end of the 21 days and is equivalent with other products.

Conclusion: Irrespective of the solvents used, the force degradation varies for different products.

\footnotetext{
${ }^{1}$ Consultant, ${ }^{2}$ Professor and Head, ${ }^{3}$ Assistant Professor ${ }^{1}$ Department of Orthodontics, Mumbai, Maharashtra, India

${ }^{2}$ Department of Orthodontics and Dentofacial Orthopedics, MA Rangoonwala College of Dental Sciences and Research Centre Pune, Maharashtra, India

${ }^{3}$ Department of Preventive Dental Sciences, Division of Orthodontics, College of Dentistry, King Khalid University, Abha Kingdom of Saudi Arabia

Corresponding Author: Rameez Asif Nachan, 644, Resham Apartments, A Wing, 1st Floor, VP Naka, 4th Nizampura Bhiwandi-421302, Maharashtra, India, e-mail: rameez_19@ hotmail.com
}

Keywords: Force degradation, Orthodontic elastomers, Tensile strength.

How to cite this article: Nachan RA, Kalia A, Al-Shahrani I. Force Degradation of Orthodontic Elastomeric Chain due to Commonly Consumed Liquids: An in vitro Study. World J Dent 2015;6(1):31-38.

\section{Source of support: Nil}

Conflict of interest: None

\section{INTRODUCTION}

Orthodontic forces deal with the closure of spaces in order to rectify the relevant malocclusion and with objectivity to achieve ideal occlusion in respective patients. The ability to close space efficiently with optimum force in orthodontic tooth movement is of major clinical importance. However, the optimal force magnitude required to close spaces orthodontically is controversial. Although the force is dependent on the root surface area of the tooth to be moved, the force magnitudes required to bodily move canines are estimated to range from as low as $100 \mathrm{~g}$ to as high as 300 or $350 \mathrm{gm}$. If, during treatment, forces fall below approximately $55 \mathrm{gm}$, bodily movement of the canines essentially ceases. Such forces needed to achieve space closure can be obtained by a number of techniques, including coil and retraction springs, closing loop arch wires, magnets and elastomeric chains. ${ }^{1-3}$

Calvin first discussed latex elastics and its use in orthodontics, since then elastics are widely used in orthodontics. Baker discussed use of these elastics to generate intermaxillary force for treating class II cases. Similar findings were reported from case and angle. Elastics are preferred mainly because of their low cost, high flexibility and resiliency. Natural rubber is an elastomer, but not all elastomers can be called 'Rubber'. The original latex is the natural sap tapped directly from the rubber tree. It contains 25 to $40 \%$ of rubber hydrocarbon (cis- 1 , 4 polyisoprene) with small amounts of protein material and fatty acid. ${ }^{2-4}$

Forces delivered by elastomeric chains are not constant and degrade overtime. The degree of degradation could result in forces that fall below the threshold value, precluding any therapeutic effect. This property of elastomeric chains has received substantial consideration over an extended period of time. However, extensive literature now exists on the properties, behavior, and modes 
of action of elastomeric chains which emphasize that thermal-cycled samples experienced less force decay than samples stored at room temperature, i.e. $37^{\circ} \mathrm{C} .{ }^{5}$ Various studies have shown a significant amount (42-75\%) of force degradation within the first 24 hours of elastomeric chain application. Other studies have also found that after this significant initial drop in the force applied, force degradation occurred at a much more stable rate. ${ }^{1-5}$

However, not many studies are available which investigated the force delivery capabilities and dimensional stability of these new colored chains under certain environmental condition with special emphasis on the variation of temperature in the oral cavity. The results obtained from these studies also prove inconsistent results and, hence, correct evaluation of degradation of force of these materials under various environmental conditions is the need of hour. ${ }^{6-9}$ Hence, an in vitro study was conducted to with the purpose to analyze and compare the effects of commonly consumed liquids, such as Cola, tea and mouthwash on the force degradation of orthodontic elastomeric chains was conducted.

\section{MATERIALS AND METHODS}

An in vitro study was done to assess factors which affect the decay properties of the elastomeric modules namely the tooth movement and temperature.

The following food stimulants were considered in the study:

- Black tea (Taj Mahal tea bags)

- Milk (Amul pasteurized homogenized full toned milk)

- Aerated drinks (CocaCola)

- Mouthwash (Dr Reddy's S-Flo containing 0.2\% sodium fluoride on aqueous base).

- Artificial saliva (it was prepared from $0.4 \mathrm{gm} \mathrm{NaCl}$, 1, $21 \mathrm{gm} \mathrm{KCl}, 0.78 \mathrm{gm} \mathrm{NaH} \mathrm{PO}_{4} \cdot 2 \mathrm{H}_{2} \mathrm{O}, 0.005 \mathrm{gm}$ $\mathrm{Na}_{2} \mathrm{~S} .9 \mathrm{H}_{2} \mathrm{O}, 1 \mathrm{gm} \mathrm{CO}\left(\mathrm{NH}_{2}\right)_{2}$ and $1000 \mathrm{ml}$ of distilled and deionized water and then $10 \mathrm{~N}$ sodium hydroxide was added).

\section{Resin Jigs ${ }^{10}$}

Polyester resin jigs $1 \mathrm{~cm}$ thick were constructed with the aid of a metal form. Four closing jigs (variable distance), each divided in half by orthodontic expansion screws and eight solid jigs (constant distance) were constructed. Fifteen pairs of stainless steel pins cut from 0.40 inch orthodontic wire were placed in rows on either side of the midline at a distance of $20 \mathrm{~mm}$ for both constant and for jig with expansion screw. The expansion screws varied the distance between the rows. Stainless steel pins were drilled in the jig with the help of micro motor and straight fissure bur and then sealed with clear cold cure acrylic material.

\section{Three Types of Elastomeric Modules were used}

1. Ormco Power Chain (product A)

2. 3M Unitek Alastik Chain (product B)

3. American Orthodontics (product C)

\section{Materials and Equipments used for Evaluating Force Decay}

1. Universal Testing Machine STS 248 (Star Testing Systems, India)

2. Metric vernier caliper

3. Electronic force gauge

Two different module lengths (long and short) of each product were used for the initial load-comparison study. Because of the size difference between the three materials, the number of units per module depended on the material. The overall unstretched length of all long and short modules was $12 \mathrm{~mm}$. By this arrangement, the long modules consisted of 3 units of product A, 4 units of product $B$, and 3 units of product $C$. The short modules consisted of 4 units of product A, 5 units of product $B$ and 4 units of product $C$. The modules were stretched $20 \mathrm{~mm}$. After stretching, the elastic chains of all 3 different products were placed on the steel pins on the jig. All the jigs containing elastic modules were then kept in a synthetic saliva medium for 15 minutes, and then thermal cycled for 30 minutes with a dwell time of 30 seconds at two different temperatures, i.e. at $45^{\circ} \mathrm{C}$ in a hot air oven and at $15^{\circ} \mathrm{C}$ in a cold temperature bath (based on work of Peterson et al. ${ }^{11}$ This study evaluated force decay over 21 days at constant extension (part I) and at decreasing extension (part II).

\section{Part I (Constant Extension)}

Forty randomly selected plastic modules from each of the three materials were randomly assigned to four groups of 10 each. Eight jigs of constant extension were used. By this arrangement, 5 long and 5 short modules of each material were tested in tea, milk, Cola and mouthwash for 10 minutes each.

\section{Part II (Decreasing Extension)}

Twenty randomly selected plastic modules from each of the three materials will be randomly assigned to four groups of 5 each. By this arrangement, 3 long and 2 short modules of each material will be tested in tea, milk, Cola and mouthwash. Four jigs of decreasing extension with expansion screw were used. The modules were stretched $20 \mathrm{~mm}$ initially (because this distance approximates the distance between the mesial wing of canine bracket and the distal wing of second premolar bracket). Later, distance was reduced by $0.5 \mathrm{~mm}$ every week with the help of a jack screw. So, the distance was reduced to $18 \mathrm{~mm} 21$ st day. 
After the jigs were immersed in particular stimulants, forces of all the elastic modules were measured on the universal testing machine. Each module on the jig was taken one at a time; force is measured and the module was kept back at its assigned place on the jig immediately. Force measurements were made at 14 time intervals: Initial, 5 minutes, 30 minutes, 1 hour, 8 hours, 24 hours, and $2,3,4,5,6,7,14$, and 21 days.

Means of percentage change in tensile strength at each follow-up with respect to initial tensile strength were calculated. $p$-values were obtained using one way analysis of variance (ANOVA) with Tukey's correction for multiple group comparisons after confirming the underlying normality assumption. $\mathrm{p}<0.05$ was considered to be statistically significant.

\section{RESULTS}

The purpose of this study was to evaluate the effect of certain liquids (Cola, tea, milk and mouthwash) on the tensile strength of the elastic chains of the products (A, $B$ and $C$ ) with long and short modules of each product at constant and variable distance. Tables 1 and 3 at constant distance and Tables 2 and 4 at variable distance gives the inter solvent comparison of percentage change in tensile strength with respect to initial tensile strength across various products for product type short and long respectively. For short product, the results were similar for product $A$ and $B$. At days 7 and 14, the average change in tensile strength is significantly smaller in tea compared to milk, Cola and mouthwash. At day 21, the average change in tensile strength is significantly smaller in tea compared to milk and mouthwash. At days 7, 14 and 21, the average change in tensile strength did not differ significantly across the solvents milk, Cola and mouthwash. Whereas for product $C$ At day 7 , the average change in tensile strength did not differ significantly across all the solvents. At days 14 and 21, the average change in tensile strength is significantly smaller in milk compared to Cola and mouthwash. For long product, the result for product A was as follows: At day 7, the average change in tensile strength is significantly higher in tea compared to milk, Cola and mouthwash. At day 14, the average change in tensile strength is significantly higher in tea compared to milk and Cola. At day 21, the average change in tensile strength did not differ significantly across the solvents milk, Cola and mouthwash. For product B, at day 7, the average change in tensile strength is significantly smaller in mouthwash compared to tea and milk and at days 14 and 21, the average change in tensile strength is significantly smaller in Cola compared to mouthwash. Whereas for product $C$, at days 7, 14 and 21, the average change in tensile strength did not differ significantly across all the solvents.

Percentage change is calculated using following formula: [(Initial tensile strength - 21 days tensile strength)]/ (initial tensile strength) $\times 100$. The inter solvent comparison of percentage change in tensile strength with respect to initial tensile strength across various products for product type long with products $\mathrm{A}, \mathrm{B}$ and $\mathrm{C}$ respectively, and Tables 5 and 7 at constant distance and Tables 6 and 8 at variable distance give the interproduct comparison

Table 1: The intersolvent comparison of percentage change in tensile strength with respect to initial tensile strength across various products (for product type: short)

\begin{tabular}{|c|c|c|c|c|c|c|c|c|c|c|}
\hline \multirow[b]{2}{*}{ Product } & \multicolumn{4}{|c|}{ Solvents } & \multicolumn{6}{|c|}{$p$-values } \\
\hline & Tea & Milk & Cola & $\begin{array}{l}\text { Mouth- } \\
\text { wash }\end{array}$ & $\begin{array}{l}\text { Tea vs } \\
\text { milk }\end{array}$ & $\begin{array}{l}\text { Tea vs } \\
\text { cola }\end{array}$ & $\begin{array}{l}\text { Tea vs } \\
\text { mouthwash }\end{array}$ & $\begin{array}{l}\text { Milk vs } \\
\text { cola }\end{array}$ & $\begin{array}{l}\text { Milk vs } \\
\text { mouthwash }\end{array}$ & $\begin{array}{l}\text { Cola vs } \\
\text { mouthwash }\end{array}$ \\
\hline \multicolumn{11}{|c|}{ Product $A$} \\
\hline Day 1 & 7.3 & 19.2 & 10.4 & 16.0 & 0.004 & 0.713 & 0.037 & 0.035 & 0.694 & 0.248 \\
\hline Day 4 & 30.8 & 45.9 & 42.4 & 46.6 & 0.001 & 0.001 & 0.001 & 0.358 & 0.984 & 0.212 \\
\hline Day 7 & 38.2 & 51.4 & 49.6 & 49.5 & 0.001 & 0.001 & 0.001 & 0.768 & 0.728 & 0.999 \\
\hline Day 14 & 46.7 & 56.9 & 54.9 & 55.2 & 0.004 & 0.022 & 0.017 & 0.860 & 0.907 & 0.999 \\
\hline Day 21 & 52.1 & 59.7 & 56.6 & 62.9 & 0.028 & 0.270 & 0.002 & 0.599 & 0.533 & 0.082 \\
\hline \multicolumn{11}{|c|}{ Product $B$} \\
\hline Day 1 & 7.0 & 14.6 & 6.3 & 13.9 & 0.001 & 0.146 & 0.001 & 0.039 & 0.993 & 0.065 \\
\hline Day 4 & 25.3 & 39.2 & 30.9 & 44.2 & 0.002 & 0.287 & 0.001 & 0.066 & 0.386 & 0.003 \\
\hline Day 7 & 34.5 & 47.4 & 47.3 & 50.0 & 0.007 & 0.007 & 0.001 & 0.999 & 0.870 & 0.849 \\
\hline Day 14 & 42.6 & 52.4 & 55.5 & 59.0 & 0.031 & 0.004 & 0.001 & 0.766 & 0.198 & 0.684 \\
\hline Day 21 & 47.8 & 55.7 & 60.5 & 62.3 & 0.039 & 0.001 & 0.001 & 0.310 & 0.101 & 0.900 \\
\hline \multicolumn{11}{|c|}{ Product C } \\
\hline Day 1 & 6.2 & 9.9 & 11.6 & 13.5 & 0.333 & 0.093 & 0.018 & 0.862 & 0.390 & 0.830 \\
\hline Day 4 & 39.2 & 34.9 & 35.9 & 41.8 & 0.150 & 0.317 & 0.513 & 0.964 & 0.010 & 0.026 \\
\hline Day 7 & 51.5 & 44.1 & 49.3 & 49.8 & 0.056 & 0.826 & 0.909 & 0.250 & 0.183 & 0.997 \\
\hline Day 14 & 55.7 & 50.8 & 56.2 & 60.4 & 0.054 & 0.989 & 0.068 & 0.030 & 0.001 & 0.118 \\
\hline Day 21 & 57.7 & 53.3 & 60.9 & 62.7 & 0.273 & 0.548 & 0.192 & 0.024 & 0.005 & 0.870 \\
\hline
\end{tabular}


Table 2: The intersolvent comparison of percentage change in tensile strength with respect to initial tensile strength across various products at variable distance (for product type: short)

\begin{tabular}{|c|c|c|c|c|c|c|c|c|c|c|}
\hline \multirow[b]{2}{*}{ Product } & \multicolumn{4}{|c|}{ Solvents } & \multicolumn{6}{|c|}{$p$-values } \\
\hline & Tea & Milk & Cola & $\begin{array}{l}\text { Mouth- } \\
\text { wash }\end{array}$ & $\begin{array}{l}\text { Tea vs } \\
\text { milk }\end{array}$ & $\begin{array}{l}\text { Tea vs } \\
\text { cola }\end{array}$ & $\begin{array}{l}\text { Tea vs } \\
\text { mouthwash }\end{array}$ & $\begin{array}{l}\text { Milk vs } \\
\text { cola }\end{array}$ & $\begin{array}{l}\text { Milk vs } \\
\text { mouthwash }\end{array}$ & $\begin{array}{l}\text { Cola vs } \\
\text { mouthwash }\end{array}$ \\
\hline \multicolumn{11}{|c|}{ Product $A$} \\
\hline Day 1 & 8.3 & 21.2 & 11.4 & 17.0 & 0.004 & 0.713 & 0.037 & 0.035 & 0.694 & 0.248 \\
\hline Day 4 & 31.8 & 47.9 & 43.4 & 47.6 & 0.001 & 0.001 & 0.001 & 0.358 & 0.984 & 0.212 \\
\hline Day 7 & 39.2 & 50.4 & 50.6 & 50.5 & 0.001 & 0.001 & 0.001 & 0.768 & 0.728 & 0.999 \\
\hline Day 14 & 47.7 & 55.9 & 54.9 & 56.2 & 0.004 & 0.022 & 0.017 & 0.860 & 0.907 & 0.999 \\
\hline Day 21 & 52.1 & 59.7 & 56.6 & 63.9 & 0.028 & 0.270 & 0.002 & 0.599 & 0.533 & 0.082 \\
\hline \multicolumn{11}{|c|}{ Product B } \\
\hline Day 1 & 7.0 & 15.1 & 6.8 & 14.4 & 0.001 & 0.146 & 0.001 & 0.039 & 0.993 & 0.065 \\
\hline Day 4 & 25.8 & 39.7 & 31.4 & 44.7 & 0.002 & 0.287 & 0.001 & 0.066 & 0.386 & 0.003 \\
\hline Day 7 & 35 & 47.9 & 47.8 & 50.5 & 0.007 & 0.007 & 0.001 & 0.999 & 0.870 & 0.849 \\
\hline Day 14 & 43.1 & 52.9 & 56 & 59.5 & 0.031 & 0.004 & 0.001 & 0.766 & 0.198 & 0.684 \\
\hline Day 21 & 48.3 & 56.2 & 61 & 62.8 & 0.039 & 0.001 & 0.001 & 0.310 & 0.101 & 0.900 \\
\hline \multicolumn{11}{|c|}{ Product C } \\
\hline Day 1 & 6.7 & 10.4 & 12.1 & 14 & 0.333 & 0.093 & 0.018 & 0.862 & 0.390 & 0.830 \\
\hline Day 4 & 39.7 & 35.4 & 36.4 & 42.3 & 0.150 & 0.317 & 0.513 & 0.964 & 0.010 & 0.026 \\
\hline Day 7 & 52 & 44.6 & 49.8 & 50.3 & 0.056 & 0.826 & 0.909 & 0.250 & 0.183 & 0.997 \\
\hline Day 14 & 56.2 & 51.3 & 56.7 & 60.9 & 0.054 & 0.989 & 0.068 & 0.030 & 0.001 & 0.118 \\
\hline Day 21 & 58.2 & 53.8 & 61.4 & 63.2 & 0.273 & 0.548 & 0.192 & 0.024 & 0.005 & 0.870 \\
\hline
\end{tabular}

Table 3: The intersolvent comparison of percentage change in tensile strength with respect to initial tensile strength across various products (for product type: long)

\begin{tabular}{|c|c|c|c|c|c|c|c|c|c|c|}
\hline \multirow[b]{2}{*}{ Product } & \multicolumn{4}{|c|}{ Solvents } & \multicolumn{6}{|c|}{$p$-values } \\
\hline & Tea & Milk & Cola & $\begin{array}{l}\text { Mouth- } \\
\text { wash }\end{array}$ & $\begin{array}{l}\text { Tea vs } \\
\text { milk }\end{array}$ & $\begin{array}{l}\text { Tea vs } \\
\text { cola }\end{array}$ & $\begin{array}{l}\text { Tea vs } \\
\text { mouthwash }\end{array}$ & $\begin{array}{l}\text { Milk vs } \\
\text { cola }\end{array}$ & $\begin{array}{l}\text { Milk vs } \\
\text { mouthwash }\end{array}$ & $\begin{array}{l}\text { Cola vs } \\
\text { mouthwash }\end{array}$ \\
\hline \multicolumn{11}{|c|}{ Product $A$} \\
\hline Day 1 & 30.1 & 15.8 & 11.2 & 19.9 & 0.001 & 0.001 & 0.022 & 0.469 & 0.550 & 0.053 \\
\hline Day 4 & 58.5 & 52.5 & 46.9 & 43.2 & 0.243 & 0.008 & 0.001 & 0.309 & 0.036 & 0.621 \\
\hline Day 7 & 61.6 & 53.3 & 49.6 & 42.4 & 0.001 & 0.001 & 0.001 & 0.102 & 0.001 & 0.001 \\
\hline Day 14 & 61.6 & 55.8 & 52.9 & 56.6 & 0.038 & 0.002 & 0.083 & 0.461 & 0.975 & 0.260 \\
\hline Day 21 & 62.6 & 59.9 & 57.4 & 61.6 & 0.563 & 0.078 & 0.962 & 0.575 & 0.834 & 0.182 \\
\hline \multicolumn{11}{|c|}{ Product B } \\
\hline Day 1 & 8.6 & 6.8 & 3.2 & 12.0 & 0.952 & 0.447 & 0.773 & 0.751 & 0.470 & 0.101 \\
\hline Day 4 & 37.7 & 46.8 & 36.5 & 41.4 & 0.112 & 0.991 & 0.763 & 0.065 & 0.489 & 0.592 \\
\hline Day 7 & 47.8 & 48.9 & 40.9 & 38.3 & 0.984 & 0.156 & 0.033 & 0.084 & 0.016 & 0.835 \\
\hline Day 14 & 47.8 & 48.9 & 43.0 & 51.5 & 0.979 & 0.356 & 0.586 & 0.199 & 0.808 & 0.040 \\
\hline Day 21 & 50.3 & 52.0 & 44.1 & 53.4 & 0.931 & 0.151 & 0.688 & 0.051 & 0.955 & 0.018 \\
\hline \multicolumn{11}{|c|}{ Product C } \\
\hline Day 1 & 10.9 & 4.8 & 5.6 & 9.4 & 0.080 & 0.153 & 0.907 & 0.983 & 0.251 & 0.419 \\
\hline Day 4 & 41.3 & 40.3 & 40.9 & 46.1 & 0.975 & 0.999 & 0.217 & 0.993 & 0.108 & 0.170 \\
\hline Day 7 & 46.4 & 46.8 & 49.9 & 49.5 & 0.995 & 0.178 & 0.265 & 0.258 & 0.369 & 0.994 \\
\hline Day 14 & 53.5 & 54.8 & 52.4 & 57.3 & 0.970 & 0.980 & 0.571 & 0.841 & 0.823 & 0.360 \\
\hline Day 21 & 56.4 & 58.0 & 54.9 & 58.1 & 0.896 & 0.900 & 0.882 & 0.525 & 0.999 & 0.505 \\
\hline
\end{tabular}

of percentage change in tensile strength at day 21 with respect to initial tensile strength across the various types of solvents for product type short and long respectively, and Graphs 1 and 3 at constant distance and Graphs 2 and 4 at variable distance. For short product, the results were similar at both constant and variable distance with the average change in tensile strength on 21 days posttreatment is significantly higher in product $\mathrm{C}$ compared to product B in tea, significantly higher in product A compared to product $C$ in milk and no difference in change in tensile strength was observed in Cola and mouthwash. For long product, the results were similar at both constant and variable distance with the average change in tensile strength on 21 days post-treatment is significantly higher in product $A$ compared to product $B$ in tea, higher in products $A$ and $C$ compared to product B in milk and Cola, whereas 
Force Degradation of Orthodontic Elastomeric Chain due to Commonly Consumed Liquids: An in vitro Study

Table 4: The intersolvent comparison of percentage change in tensile strength with respect to initial tensile strength across various products at variable distance (for product type: long)

\begin{tabular}{|c|c|c|c|c|c|c|c|c|c|c|}
\hline \multirow[b]{2}{*}{ Product } & \multicolumn{4}{|c|}{ Solvents } & \multicolumn{6}{|c|}{$p$-values } \\
\hline & Tea & Milk & Cola & $\begin{array}{l}\text { Mouth- } \\
\text { wash }\end{array}$ & $\begin{array}{l}\text { Tea vs } \\
\text { milk }\end{array}$ & $\begin{array}{l}\text { Tea vs } \\
\text { cola }\end{array}$ & $\begin{array}{l}\text { Tea vs } \\
\text { mouthwash }\end{array}$ & $\begin{array}{l}\text { Milk vs } \\
\text { cola }\end{array}$ & $\begin{array}{l}\text { Milk vs } \\
\text { mouthwash }\end{array}$ & $\begin{array}{l}\text { Cola vs } \\
\text { mouthwash }\end{array}$ \\
\hline \multicolumn{11}{|c|}{ Product $A$} \\
\hline Day 1 & 30.3 & 16 & 11.4 & 20.1 & 0.001 & 0.001 & 0.022 & 0.469 & 0.550 & 0.053 \\
\hline Day 4 & 58.7 & 52.7 & 47.1 & 43.4 & 0.243 & 0.008 & 0.001 & 0.309 & 0.036 & 0.621 \\
\hline Day 7 & 61.8 & 53.5 & 49.8 & 42.6 & 0.001 & 0.001 & 0.001 & 0.102 & 0.001 & 0.001 \\
\hline Day 14 & 61.8 & 56 & 53 & 56.8 & 0.038 & 0.002 & 0.083 & 0.461 & 0.975 & 0.260 \\
\hline Day 21 & 62.8 & 60.1 & 57.6 & 61.8 & 0.563 & 0.078 & 0.962 & 0.575 & 0.834 & 0.182 \\
\hline \multicolumn{11}{|c|}{ Product B } \\
\hline Day 1 & 8.8 & 7 & 3.4 & 12.2 & 0.952 & 0.447 & 0.773 & 0.751 & 0.470 & 0.101 \\
\hline Day 4 & 37.9 & 47 & 36.7 & 41.6 & 0.112 & 0.991 & 0.763 & 0.065 & 0.489 & 0.592 \\
\hline Day 7 & 48 & 49.1 & 41.1 & 38.5 & 0.984 & 0.156 & 0.033 & 0.084 & 0.016 & 0.835 \\
\hline Day 14 & 48 & 49.1 & 43.2 & 51.7 & 0.979 & 0.356 & 0.586 & 0.199 & 0.808 & 0.040 \\
\hline Day 21 & 50.5 & 52.2 & 44.3 & 53.6 & 0.931 & 0.151 & 0.688 & 0.051 & 0.955 & 0.018 \\
\hline \multicolumn{11}{|c|}{ Product C } \\
\hline Day 1 & 10.9 & 4.8 & 5.6 & 9.6 & 0.080 & 0.153 & 0.907 & 0.983 & 0.251 & 0.419 \\
\hline Day 4 & 41.3 & 40.3 & 40.9 & 46.3 & 0.975 & 0.999 & 0.217 & 0.993 & 0.108 & 0.170 \\
\hline Day 7 & 46.4 & 46.8 & 49.9 & 49.7 & 0.995 & 0.178 & 0.265 & 0.258 & 0.369 & 0.994 \\
\hline Day 14 & 53.5 & 54.8 & 52.6 & 57.5 & 0.970 & 0.980 & 0.571 & 0.841 & 0.823 & 0.360 \\
\hline Day 21 & 56.4 & 58.0 & 55.1 & 58.3 & 0.896 & 0.900 & 0.882 & 0.525 & 0.999 & 0.505 \\
\hline
\end{tabular}

Table 5: The interproduct comparison of percentage change in tensile strength at day 21 with respect to initial tensile strength across the various types of solvents (for product type: short)

\begin{tabular}{llllllll}
\hline & \multicolumn{3}{c}{ Product } & & \multicolumn{3}{c}{ p-values } \\
\cline { 2 - 4 } \cline { 6 - 8 } Solvent & $A$ & $B$ & $C$ & & $A$ vs B & A vs C & B vs C \\
\hline Tea & 52.1 & 47.8 & 57.7 & & 0.370 & 0.188 & 0.017 \\
Milk & 59.7 & 55.7 & 53.3 & & 0.253 & 0.046 & 0.569 \\
Cola & 56.6 & 60.5 & 60.9 & & 0.221 & 0.157 & 0.974 \\
Mouthwash & 62.9 & 62.3 & 62.7 & & 0.960 & 0.998 & 0.976 \\
\hline
\end{tabular}

it was significantly higher in products A compared to product $B$ in mouthwash.

\section{DISCUSSION}

The purpose of this study was to compare the degradation of force of orthodontic elastomeric chains on consumption of commonly consumed liquids, like tea, Cola, milk and mouthwash. For the study, samples of elastomeric modules (both short and long) of three different commercially available products were used.

Also, the module lengths of the various products (A, $B$ and $C$ ) used in this investigation were selected because they represented realistic choices that a clinician might use to retract a canine into the space of an extracted first premolar. A wide range of initial forces, in the range of 190 to 270 gm were seen, which were higher than optimal force required for orthodontic retraction (100-250 gm). To mimic the natural oral environmental condition, the products where thermal cycled and then immersed in commonly consumed liquids, like tea, milk, Cola and mouthwash.
Table 6: The interproduct comparison of percentage change in tensile strength at day 21 with respect to initial tensile strength across the various types of solvents at variable distance (for product type: short)

\begin{tabular}{llllllll}
\hline & \multicolumn{3}{c}{ Product } & & \multicolumn{3}{c}{ p-values } \\
\cline { 2 - 4 } \cline { 6 - 8 } Solvent & $A$ & $B$ & $C$ & & A vs B & A vs C & B vs C \\
\hline Tea & 51.1 & 46.8 & 56.7 & & 0.370 & 0.188 & 0.017 \\
Milk & 58.7 & 54.7 & 52.3 & & 0.253 & 0.046 & 0.569 \\
Cola & 55.6 & 59.5 & 59.9 & & 0.221 & 0.157 & 0.974 \\
Mouthwash & 61.9 & 61.3 & 61.7 & & 0.960 & 0.998 & 0.976 \\
\hline
\end{tabular}

Table 7: The interproduct comparison of percentage change in tensile strength at day 21 with respect to initial tensile strength across the various types of solvents (for product type: long)

\begin{tabular}{llllllll}
\hline & \multicolumn{3}{c}{ Product } & & \multicolumn{3}{c}{$p$-values } \\
\cline { 2 - 4 } \cline { 6 - 8 } Solvent & $A$ & $B$ & $C$ & & A vs B & A vs C & $B$ vs C \\
\hline Tea & 62.6 & 50.3 & 56.4 & & 0.007 & 0.189 & 0.194 \\
Milk & 59.9 & 52.0 & 58.0 & & 0.005 & 0.609 & 0.027 \\
Cola & 57.4 & 44.1 & 54.9 & & 0.001 & 0.404 & 0.001 \\
Mouthwash & 61.6 & 53.4 & 58.1 & & 0.004 & 0.229 & 0.091 \\
\hline
\end{tabular}

Table 8: The interproduct comparison of percentage change in tensile strength at day 21 with respect to initial tensile strength across the various types of solvents at variable distance (for product type: long)

\begin{tabular}{llllllll}
\hline & \multicolumn{3}{c}{ Product } & & \multicolumn{3}{c}{$p$ values } \\
\cline { 2 - 4 } \cline { 6 - 8 } Solvent & $A$ & $B$ & $C$ & & $A$ vs B & $A$ vs C & $B$ vs C \\
\hline Tea & 62.1 & 49.8 & 55.9 & & 0.007 & 0.189 & 0.194 \\
Milk & 59.5 & 51.5 & 57.5 & & 0.005 & 0.609 & 0.027 \\
Cola & 56.9 & 43.6 & 54.4 & & 0.001 & 0.404 & 0.001 \\
Mouthwash & 61.1 & 52.9 & 57.6 & & 0.004 & 0.229 & 0.091 \\
\hline
\end{tabular}

There was significant loading force degradation within the first 24 hours upto day 7 for all three products in the solvents used. Similar results were seen in other 


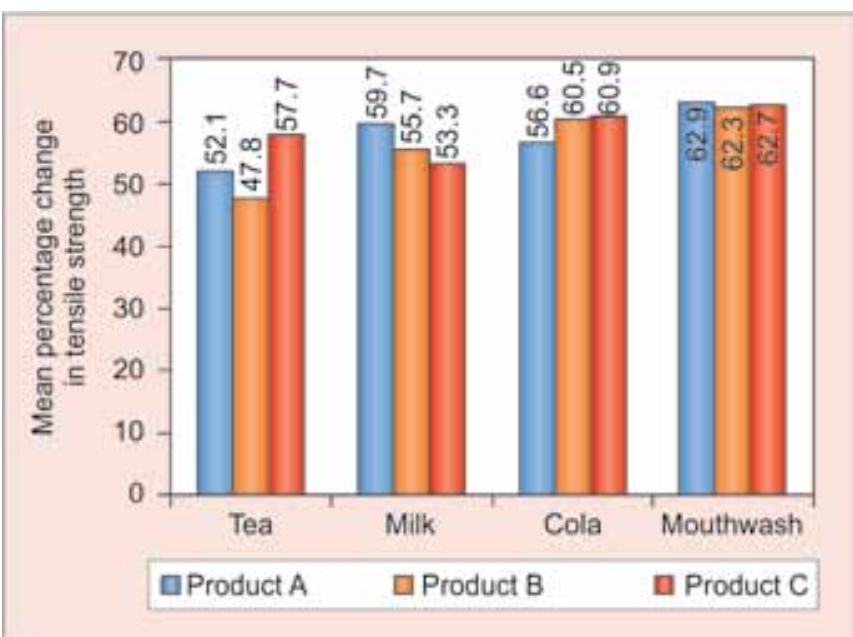

Graph 1: The interproduct comparison of percentage change in tensile strength at day 21 with respect to initial tensile strength across the various types of solvents (for product type: short)

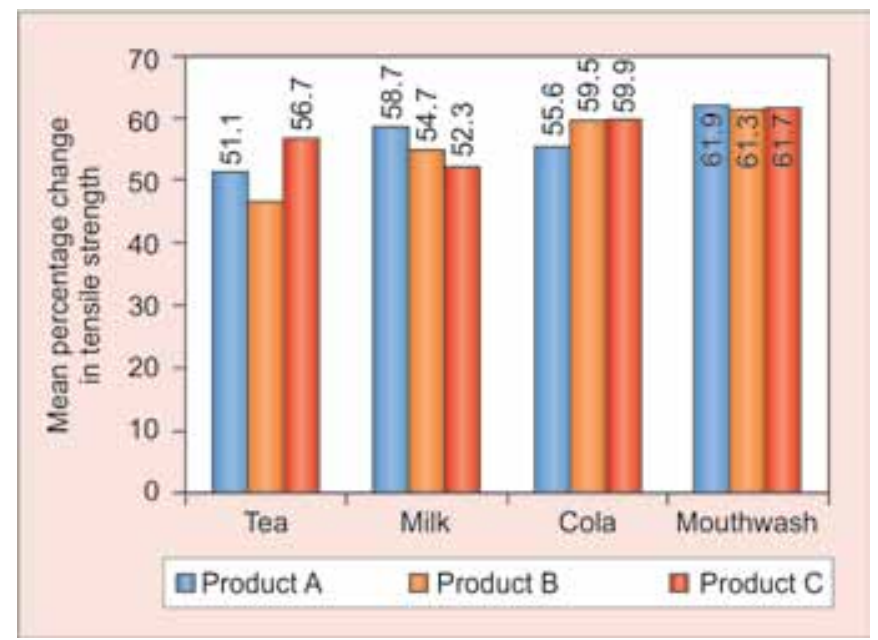

Graph 3: The interproduct comparison of percentage change in tensile strength at day 21 with respect to initial tensile strength across the various types of solvents at variable distance (for product type: short)

studies. ${ }^{14,6,12,13}$ The remaining force exerted was within the range for optimal tooth movement (170-250 gm).

Between days 1-4, 7-14 and 14-21, the force decay curves for the three products (A, B and C) kept immersed in different solvents were found to be gradually reducing suggesting that significant force degradation occurred from day 7 to 14 day. ${ }^{1,2,6-9,11}$ This suggests that the tensile strength of both short and long type of elastomeric products available, significantly reduced after 1 week for all the three products (A, B and C).

\section{Comparison of Percentage Change in Tensile Strength of All Products with All Solvents in Product Type Short}

\section{Product A (Constant and Variable Distance)}

While evaluating the force degradation in percentage for short type of elastomeric modules, product A seems

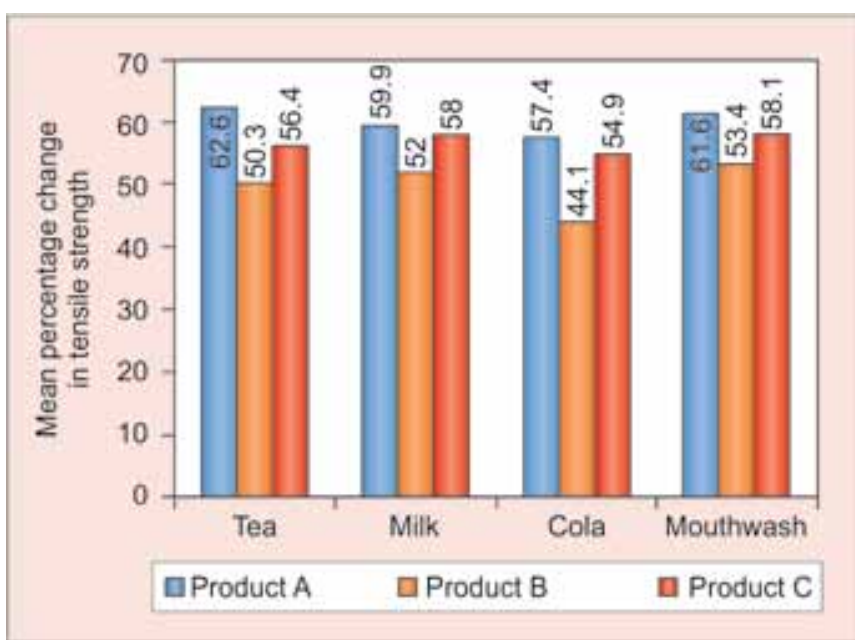

Graph 2: The interproduct comparison of percentage change in tensile strength at day 21 with respect to initial tensile strength across the various types of solvents (for product type: long)

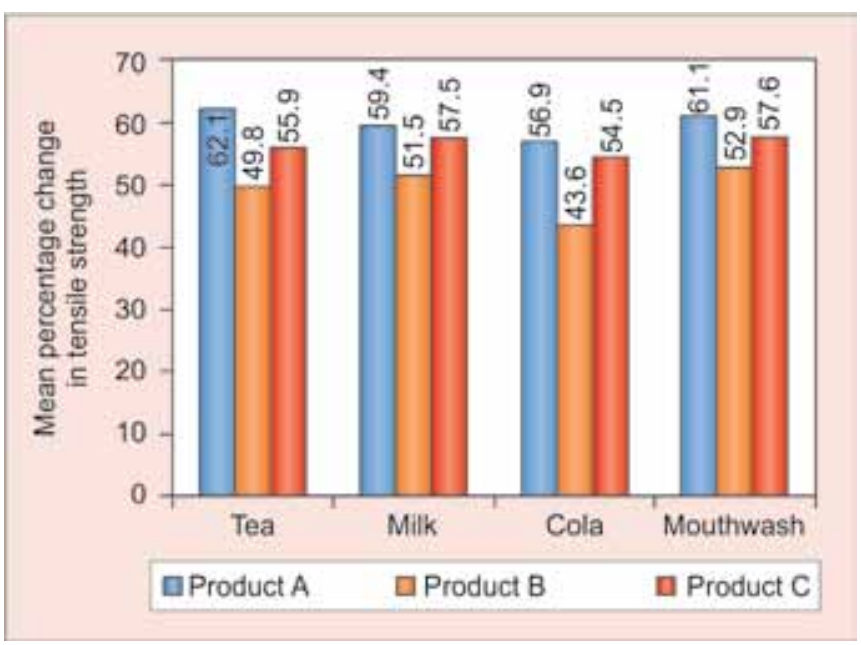

Graph 4: The interproduct comparison of percentage change in tensile strength at day 21 with respect to initial tensile strength across the various types of solvents at variable distance (for product type: long)

to vary in force degradation with respect to different solvents at different time intervals. However, it was significantly higher in tea as compared to other solvents.

\section{Product B (Constant and Variable Distance)}

While evaluating the force degradation in percentage for short type of elastomeric modules, product B seems to vary in force degradation with respect to different solvents at different time intervals. However, it was significantly higher in tea as compared to other solvents.

\section{Product C (Constant and Variable Distance)}

While evaluating the force degradation in percentage for short type of elastomeric modules, product $C$ seems to vary in force degradation with respect to different solvents at different time intervals. However, it was significantly higher in milk as compared to other solvents. 


\section{Comparison of Percentage Change in Tensile Strength of All Products with All Solvents in Product Type Long}

\section{Product A (Constant and Variable Distance)}

While evaluating the force degradation in percentage for short type of elastomeric modules, product A seems to vary in force degradation with respect to different solvents at different time intervals. However, it was significantly higher in tea as compared to other solvents.

\section{Product B (Constant and Variable Distance)}

While evaluating the force degradation in percentage for short type of elastomeric modules, product B seems to vary in force degradation with respect to different solvents at different time intervals. However, it was significantly higher in Cola as compared to other solvents.

\section{Product C (Constant and Variable Distance)}

While evaluating the force degradation in percentage for short type of elastomeric modules, product $C$ seems to vary in force degradation with respect to different solvents at different time intervals. However, it was significantly higher in milk as compared to other solvents.

\section{Comparison of Percentage Change in Tensile Strength of All Products with All Solvents at Day 21}

In short product type (constant and variable distance):

1. The evaluated force degradation in percentage in tensile strength on 21 days post-treatment in tea was significantly higher in product $C$ compared to products $\mathrm{A}$ and $\mathrm{B}$ and slightly more significant when the distance was reduced.

2. The evaluated force degradation in percentage in tensile strength on 21 days post-treatment in milk was significantly higher in product A compared to products $B$ and $C$ and slightly more significant when the distance was reduced.

3. The evaluated force degradation in percentage in tensile strength on 21 days post-treatment in Cola and mouthwash solvents did not differ significantly across three products.

In long product type (constant and variable distance):

1. The evaluated force degradation in percentage in tensile strength on 21 days post-treatment in tea was significantly higher in product A compared to products $\mathrm{B}$ and $\mathrm{C}$.

2. The evaluated force degradation in percentage in tensile strength on 21 days post-treatment in milk was significantly higher in products $\mathrm{A}$ and $\mathrm{C}$ as compared to product $B$.

3. The evaluated force degradation in percentage in tensile strength on 21 days post-treatment in Cola was significantly higher in products $\mathrm{A}$ and $\mathrm{C}$ as compared to product $B$.

4. The evaluated force degradation in percentage in tensile strength on 21 days post-treatment in mouthwash was significantly higher in products $\mathrm{A}$ and $\mathrm{C}$ as compared to product $\mathrm{B}$.

As expected, the short modules produced significantly higher force level throughout the 21 days test period compared to longer samples of each materials. At the end of 21 days, product A's short and long samples produced lowest mean force levels as compared to products $B$ and product $C$. The rate of tooth movement used in this study to determine its effect on force decay was based on investigation by Boester and Johnston. ${ }^{14}$ Their data indicates that, $0.5 \mathrm{~mm}$ per week is a reasonable rate of tooth movement into an extraction site. According to their study, reduction of length over which the modules where stretched to stimulate tooth movement had a highly significant influence over the amount of force available at 14 and 21 days compared to those modules held at constant length.

However, our study did not show significant difference in force available at 14 and 21 days in variable length and constant length group. The limitation of this study was that it is an in vitro evaluation which does not give predictable results as compared to in vivo studies. On an average, the force decay after 21 days, for product $\mathrm{B}$ was better as compared to products $\mathrm{C}$ and $\mathrm{A}$ in all the consumed liquids.

\section{CONCLUSION}

From this in vitro study, we conclude that:

- The evaluated force degradation in percentage in tensile strength on 21 days post-treatment in tea was significantly higher in product A compared to products $\mathrm{B}$ and $\mathrm{C}$ with product type short.

- The evaluated force degradation in percentage in tensile strength on 21 days post-treatment in Cola was significantly higher in products $\mathrm{A}$ and $\mathrm{C}$ as compared to product $B$ with product type short

- The force degradation of Product B was significantly less compared to the products $\mathrm{A}$ and $\mathrm{C}$ initially. However, it degrades at the end of the 21 days and is equivalent with other products.

- Therefore, we can conclude that, irrespective of the solvents used, the force degradation varies for different products. 


\section{REFERENCES}

1. Josell SD, Leiss JL, Rekow ED. Force degradation in elastomeric chains. Semin Orthod 1997;3(3):189-197.

2. Singh VP, Pokhrael PR, Pariekh K, Roy DK. Elastics in orthodontics: a review. Health Renaissance 2012;10(1): 49-56.

3. Balhoff DA, Shuldberg M, Hagan JL, Ballard RW, Armbruster PC. Force decay of elastomeric chains-a mechanical design and product comparison study. J Orthod 2011;38(1):40-47.

4. Fernandes DJ, Abrahao GM, Artese F, Elias CN and Mende AM. Force relaxation characteristics of medium force orthodontic latex elastics: A pilot study. ISRN Dentistry 2011;2011: 536089.

5. Eliades T, Eliades G, Silika N, Watts DC. Tensile properties of orthodontic elastomeric chains. Eur J Orthod 2004;26(2):157-162.

6. Andreasen GF, Bishara S. Comparison of alastic chains with elastic involved with intra-arch molar to molar forces. Angle Orthod 1970;40(3):151-158.

7. Kuster R, Ingervall B, Biirgrn W. Laboratory and intraoral tests of the elastic chains. Eur J Orthod 1986;8(3):202-208.
8. Baty DL, Volz JE, von Fraunhofer JA. Force delivery properties of colored elastomeric modules. Am J Orthod Dentofac Orthop 1994;106(1):40-46.

9. Kanchana P, Godfrey K. Calibration of force extension and force degradation characteristics of orthodontic latex elastics. Am J Orthod Dentofacial Orthop 2000;118(3):280-287.

10. De Genova DC, Mclnnes-Ledoux P, Weinberg R, Shaye R. Force degradation of orthodontic elastomeric chains-a product comparison study. Am J Orthod Dentofac Orthop 1985;87(5):377-384.

11. Peterson EA II, Philips RW, Schwartz ML. A comparison of the physical properties of four restorative resins. J Am Dent Assoc 1966;73(6):1324-1336.

12. Hershey G, Reynolds WG, Hill C. The plastic module as an orthodontic tooth-moving mechanism. Am J Orthod 1975;67(5):554-562.

13. Ash JL, Nikolai RJ. Relaxation of orthodontic elastomeric chains and modules in vitro and in vivo. J Dent Res 1978; 57(5-6):685-690.

14. Boester Ch, Jhonston LE. A clinical investigation of the concepts of differential and optimal force in canine retraction. Angle Orthod 1974;44(2):113-119. 